\title{
Sound emissions by a laboratory bubble cloud
}

\author{
M. Nicholas, a) R. A. Roy, ${ }^{\text {b) }}$ and L. A. Crum ${ }^{\text {b) }}$ \\ National Center for Physical Acoustics, The University of Mississippi, University, Mississippi 38677 \\ H. Oguz and A. Prosperetti \\ Department of Mechanical Engineering, The Johns Hopkins University, Baltimore, Maryland 21218
}

(Received 20 August 1992; accepted for publication 25 January 1994)

\begin{abstract}
This paper presents the results obtained from a detailed study of the sound field within and around a cylindrical column of bubbles generated at the center of an experimental water tank. The bubbles were produced by forcing air through a circular array of hypodermic needles. As they separated from the needles the "birthing wails" produced were found to excite the column into normal modes of oscillation whose spatial pressure-amplitude distribution could be tracked in the vertical and horizontal directions. The frequencies of vibration were predicted from theoretical calculations based on a collective oscillation model and showed close agreement with the experimentally measured values. On the basis of a model of the column excitation, absolute sound levels were analytically calculated with results again in agreement with the measured values. These findings provide considerable new evidence to support the notion that bubble plumes can be a major source of underwater sound around frequencies of a few hundred hertz.
\end{abstract}

PACS numbers: 43.30.Ft, 43.30.Nb, 43.30.Jx

\section{INTRODUCTION}

Wave breaking is the most significant air-entrainment process occurring at the surface of the ocean and gives rise to a large number of bubble clouds (Thorpe, 1982, 1986; Thorpe and Hall, 1983; Monahan and Mac Niocaill, 1986; Monahan and $\mathrm{Lu}, 1990$ ). It is known that the number and intensity of breaking waves are strongly dependent on the wind speed above the ocean surface (see, e.g., Toba and Koga, 1986; Phillips, 1988; Wu, 1988), and it has also been shown that a correlation exists between wind speed and the intensity of low-frequency (below $1 \mathrm{kHz}$ ) ambient sound in the ocean (Wenz, 1962; Piggott, 1964; Perrone, 1969; Kerman, 1984; Kuperman and Ferla, 1985; Wille and Geyer, 1985; Carey and Wagstaff, 1986; Kennedy and Goodnow, 1990; Kewley et al., 1990; Kennedy, 1992). These circumstances have led to the consideration of processes by which breaking waves may produce such lowfrequency noise (Wilson, 1980; Kerman, 1984). Carey and co-workers (Carey and Bradley, 1985; Carey and Browning, 1988) and, independently, Prosperetti (1985, 1988a, 1988b) have suggested that collective oscillations of the bubble clouds produced by breaking waves could be responsible for the low-frequency emissions. The argument was essentially that, since the bubbles in the cloud constitute a collection of coupled oscillators, one would expect the existence of normal modes of oscillation of the cloud itself at frequencies far lower than the frequency of oscillation of the individual bubbles. This idea has been explored in, and supported by, a number of subsequent pub-

\footnotetext{
a) Present address: Naval Research Laboratory, Code 7140, 4555 Overlook Ave. S.W., Washington, DC 20375-5350.

${ }^{b}$ Present address: Applied Physics Laboratory, University of Washington, Seattle, WA 98105.
}

lications (Lu et al., 1990; Yoon et al., 1991; Carey et al., 1993; Prosperetti et al., 1993; Koller and Shankar, 1993; O $\widetilde{g} u z, 1994)$.

In view of the difficulty in gathering oceanic field data on the role of bubble clouds in low-frequency sound generation, the conclusions mentioned above rest mainly on theoretical analyses only partially verified in laboratory experiments. It is therefore important to validate further the theoretical models used so as to gain confidence in their predictions. Initial experiments (Yoon et al., 1991) have shown that bubble clouds are capable of collective oscillations at frequencies far below those of the individual constituent bubbles in excellent agreement with theory. In this paper that work is extended in two significant ways. In the first place, the use of a much more extensive data set renders the measurement of the higher mode frequencies possible with a very good match with theory. Second, the model is extended to the prediction of the absolute acoustic levels, again in good agreement with data. This is a very nontrivial point as it presupposes a quantitative understanding of the mechanism by which the bubble cloud is excited. Our conclusion is that the energy imparted to the individual bubbles upon their formation coupled with the spectral width of single-bubble free oscillations accounts for the level of acoustic radiation observed in the experiment. In a separate study (O $\widetilde{\mathrm{guz}}, 1994$ ), it is shown that, on the same basis, good predictions of low-frequency oceanic ambient noise can be found. These successful estimates of levels are a stringent test of the theory as it is well known that, in general, it is much easier to match frequencies than levels.

\section{EXPERIMENTAL PROCEDURE}

The experimental arrangement used in this work is very similar to the one described in Yoon et al. (1991) and 


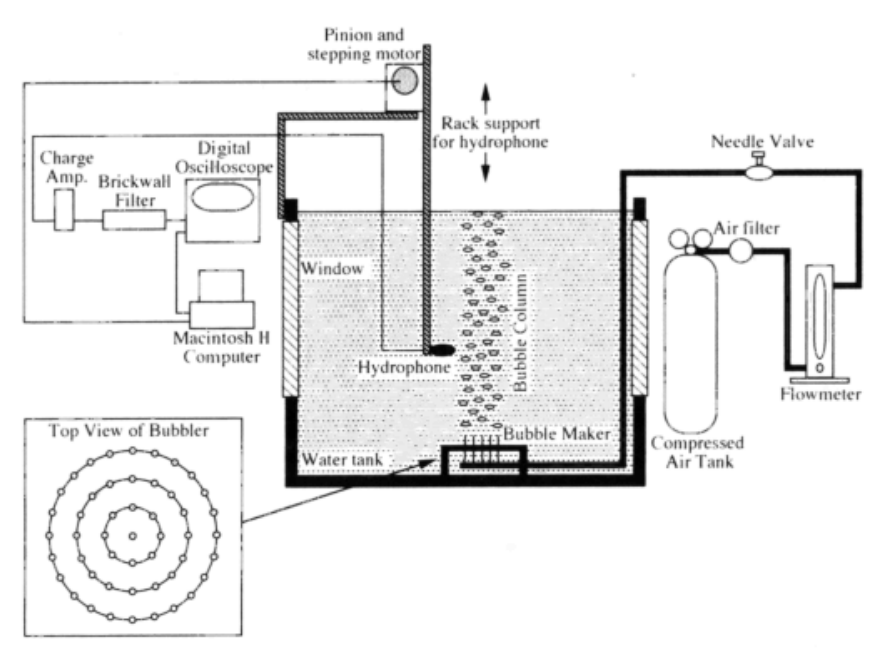

FIG. 1. Schematic diagram of the experimental apparatus used to measure the sound emissions from the higher-order modes of a bubble column.

consisted of three concentric rings of 22-gauge needles at the bottom of a $1 \times 1 \times 1 \mathrm{~m}^{3}$ Plexiglas-and-wood tank filled with tap water. A schematic diagram of the apparatus is shown in Fig. 1.

The gas volume fraction in the column $\beta$ (i.e., the fraction of the total volume occupied by the gas) was calculated from

$$
\beta=t \dot{V} / S h \text {, }
$$

where $\dot{V}$ is the precisely metered volume of air entering the column per unit time, $t$ is the rise time of the bubbles, $S$ is the cross-sectional area of the column, and $h$ is the water depth. Because of the slow recirculating flow set up within the column, the bubbles at the periphery have a rise time somewhat longer than those in the center. The difference was measured to be of the order of $14 \%$ and an average value was used. Another effect of the recirculating flow is a slight upward broadening of the bubble column the average diameter of which (uniform within 10\%) was measured to be $70 \mathrm{~mm}$. The experimental accuracy on the average value of $\beta$ is judged to be $\pm 10^{-4}$.

Figure 2 shows a photograph of the bubbles within the column for a void fraction of $1.05 \%$. The shape is somewhat flattened and spheroidal, but the volume can be estimated by measuring the semimajor and semiminor axes. From 300 such measurements (150 bubbles each for $\beta=0.70 \%$ and $1.05 \%$ ), we find the equivalent radius distribution shown in Fig. 3. The mean equivalent radius is $1.8 \pm 0.2 \mathrm{~mm}$ so that the assumption of a monodisperse mixture used in the theory described in the next section is justified.

The plywood and Plexiglas walls are essentially "soft" (i.e., pressure-release) at the frequencies considered. Since the tank was elevated off the floor by means of two wooden beams along two opposite edges of its base, the same may be assumed for this boundary as well. In order to also obtain data with a different bottom boundary condition, a 5-cm-thick concrete slab was constructed to cover the entire bottom of the tank. An array of holes exactly

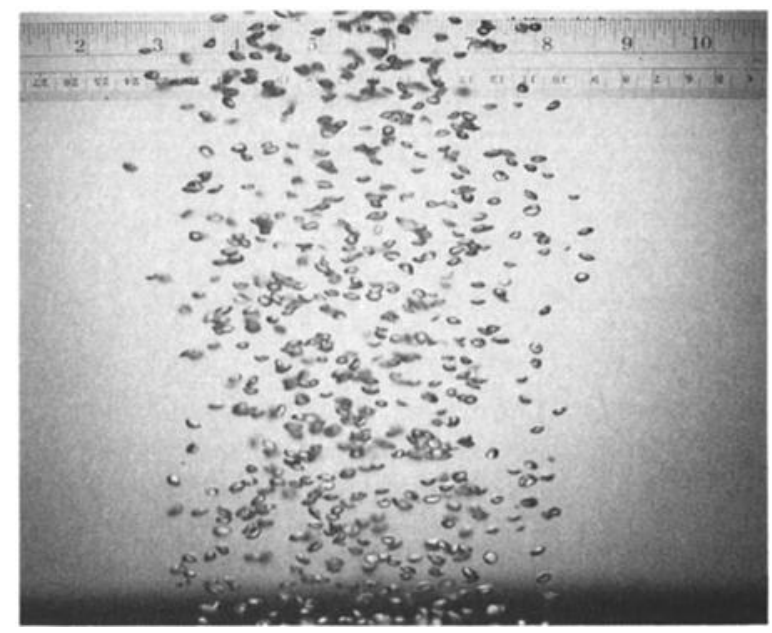

FIG. 2. Photograph of the bubbles in a column with a $1.05 \%$ volume fraction. The averaged bubble radius measured over 150 bubbles was $1.9 \pm 0.1 \mathrm{~mm}$ for this void fraction.

matching the location of the ports of the bubble maker was made in the center of this slab so that the needle tips would be flush with the surface of the concrete boundary.

The position of the receiver in the tank could be accurately set by using a rack-and-pinion arrangement driven by a computer-controlled precision stepping motor. At each hydrophone location the average power spectrum of the column noise was measured and the average over 500 FFT spectra calculated. (Some of the earlier measurements were averaged over 1000 FFT spectra, but the slight improvement over 500 averages was not thought to be worth the extra investment in time). A 625-point FFT was used with a Nyquist frequency of $2.5 \mathrm{kHz}$. The sampling time interval was $0.1 \mathrm{~s}$ and the frequency resolution was therefore $10 \mathrm{~Hz}$. In order to establish a value for the experimental uncertainty we took either five or ten sets of

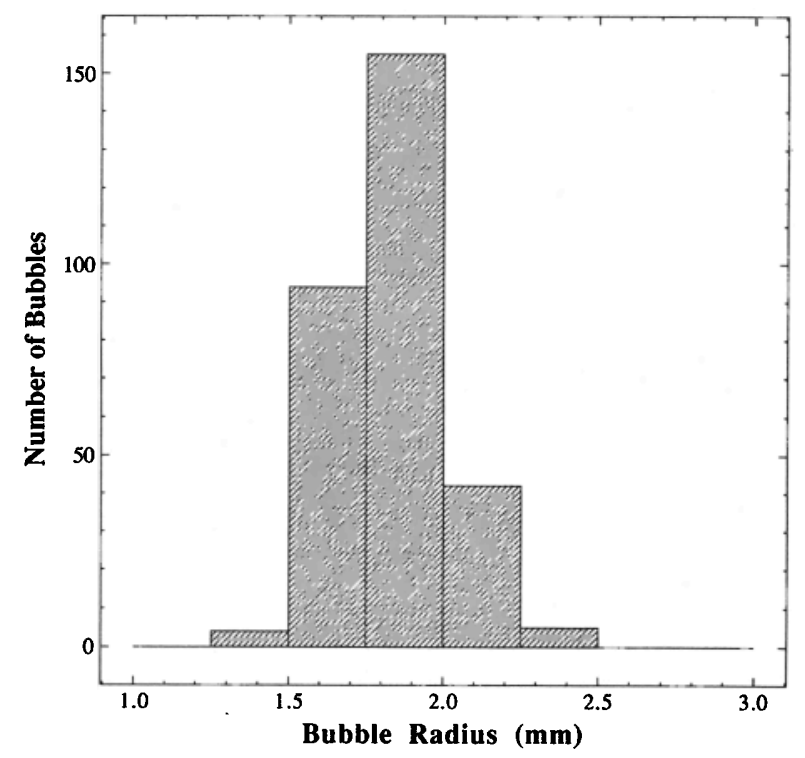

FIG. 3. Bubble size distribution obtained from 300 bubbles, 150 from a column with $\beta=1.05 \%$ and 150 from a column with $\beta=0.70 \%$. 
measurements at each hydrophone location. The standard deviation was found to be of the order of $1 \mathrm{~dB}$ and is too small to be shown in the figures to be discussed below.

In examining the power spectra of the measured sound field within the tank, it was noted that the measured values rarely fell below roughly $70 \mathrm{~dB}$ re: $1 \mu \mathrm{Pa} / \mathrm{Hz}^{1 / 2}$. This must therefore be considered the effective "noise floor" for the measurements.

\section{MATHEMATICAL FORMULATION}

To model the experimental arrangement described in Sec. I, we consider a circular column of bubbly liquid of radius $R_{c}$ concentric with a circular tank of radius $R_{t}>R_{c}$. While this model reduces the number of spatial variables to be considered and substantially simplifies the analysis, it clearly fails to match the actual geometry of the experiment. The error introduced thereby is important in certain parameter ranges and will be analyzed later.

As for the excitation of the modes of the system, there are only a few possibilities: One is the buoyant ascensional motion of the bubbles, possible vortex shedding, and wake interactions. Characteristic frequencies associated with these processes are at most a few hertz, and it appears unlikely that they can have an effect at frequencies of the order of $0.5-1 \mathrm{kHz}$. Arrangements like the one described are known to be subject to a three-dimensional instability when the rise time of the bubbles is comparable to the period of standing surface waves in the tank. The associated frequencies are even lower, at most of the order of fractions of hertz. The only remaining energetic process is the formation and pinching off of the bubbles from the needles and indeed it is observed in the experiment that, immediately after shutting off the gas flow, the noise level falls to the background level even though all other conditions in the column are virtually unchanged. The frequency mismatch between single-bubble oscillations and the modes of the column is of one order of magnitude, and the possibility of energy transfer is, as will be shown, much more likely. In the model we assume therefore that a timeharmonic, spatially uniform forcing acts at the base of the column of bubbly liquid.

In presenting the theoretical framework for the simulation of the present experiment, we separate the description of the system from that of the forcing.

\section{A. The physical system}

Since the problem is linear, we assume that the system is forced by an imposed pressure field of unit amplitude and time dependence $\exp (i \omega t)$ at the base of the column of bubbly liquid. Upon separation of the time variable, the disturbance pressure field in the pure liquid, $P$, satisfies Helmholtz's equation with the wave number

$$
k=\omega / c \text {, }
$$

where $c$ is the speed of sound in the liquid. As shown in several earlier publications (see, e.g., Commander and Prosperetti, 1989), the disturbance pressure field in the bubbly mixture, $\boldsymbol{P}^{(m)}$, satisfies Helmholtz's equation with the wave number

$$
\kappa^{2}=\frac{\omega^{2}}{c^{2}}+4 \pi \omega^{2} \frac{n a}{\omega_{0}^{2}-\omega^{2}+2 i b \omega} .
$$

Here $n$ is the number of bubbles per unit volume, $a$ their (equilibrium) radius, and $\omega_{0}$ and $b$ the (frequencydependent, effective) natural frequency and damping parameter of the individual bubbles. In writing this equation it has been assumed that all bubbles have the same radius. As explained above, this is fairly well justified in the present experiment.

As in earlier papers (Lu et al., 1990; Yoon et al., 1991; Lu and Prosperetti, 1993; Prosperetti et al., 1993; Sarkar and Prosperetti, 1993) the transition from the bubbly mixture to the pure liquid in the tank is assumed to occur at a geometrical surface $r=R_{c}$ across which pressure and velocity (or, at the present small gas volume fractions, pressure and pressure gradient) are continuous. The cylindrical radial coordinate $r$ is measured from the common axis of the cylindrical surfaces that define the boundaries of the system. After separation of the time dependence, the pressure disturbance at the base of the column has the value

$$
P^{(m)}(r, z=-h)=1, \quad 0 \leqslant r \leqslant R_{c},
$$

where $h$ is the height of the column. The free surface $z=0$ of the tank acts as a pressure release boundary so that

$$
P(r, z=0)=0, \quad 0 \leqslant r<R_{t} .
$$

In our earlier studies (Lu et al., 1990; Yoon et al., 1991) an excellent agreement between theory and experiment had been obtained by assuming that both the bottom and the side walls of the tank behaved as pressure-release boundaries. Here we shall make the same assumption for the side walls,

$$
P\left(r=R_{t}, z\right)=0, \quad-h<z \leqslant 0,
$$

and for the experimental situation without the concrete slab inserted at the bottom of the tank,

$$
P(r, z=-h)=0, \quad R_{c}<r \leqslant R_{t}, \quad \text { "soft" bottom. }
$$

With the concrete slab in place, we assume instead a rigid boundary condition:

$$
\left.\frac{\partial P}{\partial z}\right|_{z=-h}=0, \quad R_{c}<r \leqslant R_{t}, \quad \text { "rigid" bottom. }
$$

The validity of these models can also be examined experimentally, as will be discussed below.

Outside the bubbly mixture the general solution of the Helmholtz equation satisfying (5), (6), and either (7) or (8) may be written

$$
P=\sum_{n=1}^{\infty} C_{n} f_{n}(r) \sin \lambda_{n} z
$$

Here, for the soft-bottom case, we take

$$
\lambda_{n} \equiv \lambda_{n}^{S}=\pi n / h, \quad \text { "soft" bottom, }
$$

while, for the rigid-bottom case,

$$
\lambda_{n} \equiv \lambda_{n}^{R}=(\pi / h)\left(n-\frac{1}{2}\right), \quad \text { "rigid" bottom. }
$$


The functions $f_{n}(r)$ can be written in several equivalent ways, each of which presents some numerical advantages in different ranges of the variables. We use the following forms:

$$
\begin{aligned}
f_{n}(r)= & \frac{J_{0}\left(r \mu_{n}\right)}{J_{0}\left(R_{t} \mu_{n}\right)}-\frac{Y_{0}\left(r \mu_{n}\right)}{Y_{0}\left(R_{t} \mu_{n}\right)}, \quad \mu_{n} \text { real, } \\
= & H_{0}^{(1)}\left(r \mu_{n}\right) \\
& -\frac{H_{0}^{(1)}\left(R_{t} \mu_{n}\right)}{J_{0}\left(R_{t} \mu_{n}\right)} J_{0}\left(r \mu_{n}\right), \quad \mu_{n} \text { imaginary, }
\end{aligned}
$$

where

$$
\mu_{n}=\sqrt{k^{2}-\lambda_{n}^{2}}
$$

To express the pressure disturbance $P^{(m)}$ in the bubbly liquid we use superposition and write

$$
P^{(m)}=-\frac{\sin \kappa z}{\sin \kappa h}+\sum_{k=1}^{\infty} A_{k} J_{0}\left(r \mu_{k}^{(m)}\right) \sin \lambda_{k}^{S},
$$

where

$$
\mu_{k}^{(m)}=\sqrt{\kappa^{2}-\left(\lambda_{k}^{S}\right)^{2}} .
$$

The first term is a solution of the Helmholts equation satisfying the free-surface boundary condition (5) and the bottom condition (4). The second term satisfies the same free-surface condition, but vanishes at the bottom $z=-h$. Since this is the same bottom condition applicable to the pure liquid region in the soft case, $\lambda_{k}=\lambda_{k}^{S}$ in (15). Rather than using superposition as in (15), the standard approach to this problem would be to take a Hankel transform. The result is the same, but the method much more cumbersome than the present one.

The coefficients $A_{k}$ and $C_{n}$ are to be determined by imposing the continuity requirements at $r=R_{c}$ mentioned before. The condition of continuity of pressure gives

$$
\sum_{n=1}^{\infty} A_{n} J_{0}\left(x_{n}\right) \sin \lambda_{n}^{S} z-\sum_{n=1}^{\infty} C_{n} f_{n}\left(R_{c}\right) \sin \lambda_{n} z=\frac{\sin \kappa z}{\sin \kappa h} .
$$

Similarly, the continuity of the pressure derivative normal to $r=R_{c}$ gives

$$
\sum_{n=1}^{\infty} A_{n} x_{n} J_{1}\left(x_{n}\right) \sin \lambda_{n}^{S} z-\sum_{n=1}^{\infty} C_{n} s_{n} g_{n}\left(R_{c}\right) \sin \lambda_{n} z=0,
$$

where $x_{n}=R_{d} \mu_{n}^{(m)}, s_{n}=R_{d} \mu_{n}$, and, depending on the form of $f_{n}$ used, $g_{n}$ is given by one of the expressions

$$
g_{n}(r)=\frac{J_{1}\left(R_{d} \mu_{n}\right)}{J_{0}\left(R_{t} \mu_{n}\right)}-\frac{Y_{1}\left(R_{d} \mu_{n}\right)}{Y_{0}\left(R_{t} \mu_{n}\right)}, \quad \mu_{n} \text { real, }
$$

or

$$
\begin{aligned}
g_{n}(r)= & H_{1}^{(1)}\left(R_{d} \mu_{n}\right) \\
& -\frac{H_{0}^{(1)}\left(R_{t} \mu_{n}\right)}{J_{0}\left(R_{t} \mu_{n}\right)} J_{1}\left(R_{d} \mu_{n}\right), \quad \mu_{n} \text { imaginary. }
\end{aligned}
$$

In view of the completeness of the set $\left\{\sin \lambda_{n} z\right\}$ for both the soft- and the rigid-bottom cases the coefficients $\left\{A_{n}\right\}$ and $\left\{C_{n}\right\}$ can be determined by taking the scalar product of (17) and (18) with $\sin \lambda_{j} z, j=1,2, \ldots$. In this way, and assuming that the order of integration and summations can be interchanged, one finds

$$
\begin{aligned}
& \sum_{n=1}^{\infty} a_{j n} J_{0}\left(x_{n}\right) A_{n}-\frac{1}{2} f_{n}\left(R_{c}\right) C_{j}=b_{j}, \\
& \sum_{n=1}^{\infty} a_{j n} x_{n} J_{1}\left(x_{n}\right) A_{n}-\frac{1}{2} s_{j} g_{j}\left(R_{c}\right) C_{j}=0 .
\end{aligned}
$$

Here

$$
\begin{aligned}
a_{j n} & =\int_{-h}^{0} \sin \lambda_{j} z \sin \lambda_{n}^{(m)} z d z, \\
b_{j} & =\frac{1}{\sin \kappa h} \int_{-h}^{0} \sin \lambda_{j} z \sin \kappa z d z \\
& =\frac{1}{\sin \kappa h}\left(\frac{\sin \left(\kappa-\lambda_{j}\right) h}{\left(\kappa-\lambda_{j}\right) h}-\frac{\sin \left(\kappa+\lambda_{j}\right) h}{\left(\kappa+\lambda_{j}\right) h}\right) .
\end{aligned}
$$

Upon elimination of the $C_{j}$ 's the following infinite algebraic system for the $A_{n}$ 's is found:

$$
\sum_{n=1}^{\infty}\left(J_{0}\left(x_{n}\right)-\frac{x_{n} J_{1}\left(x_{n}\right) f_{j}\left(R_{c}\right)}{s_{j} g_{j}\left(R_{c}\right)}\right) a_{j n} A_{n}=b_{j} .
$$

For the soft-bottom case, $\lambda_{j}=\lambda_{j}^{S}$ and $a_{j n}=\frac{1}{2} \delta_{j n}$, so that the solution of the system $(25)$ is

$$
A_{n}=b_{n}\left(J_{0}\left(x_{n}\right)-\frac{x_{n} J_{1}\left(x_{n}\right) f_{n}\left(R_{c}\right)}{s_{n} g_{n}\left(R_{c}\right)}\right)^{-1} .
$$

For the rigid-bottom case, however,

$$
a_{j n}=\frac{\sin \left(\lambda_{j}^{R}-\lambda_{n}^{S}\right) h}{\left(\lambda_{j}^{R}-\lambda_{n}^{S}\right) h}-\frac{\sin \left(\lambda_{j}^{R}+\lambda_{n}^{S}\right) h}{\left(\lambda_{j}^{R}+\lambda_{n}^{S}\right) h},
$$

and it is necessary to find an approximate solution numerically after truncation to a finite number of equations. It is found that the coefficient matrix is well behaved and any standard method of solution can be used. We have adopted the $\mathrm{L}-\mathrm{U}$ decomposition technique retaining 30 terms in the sum (25). This number was established on the basis of the usual convergence tests.

As a check on both the analysis and the numerical results, in the rigid-bottom case, one can take scalar products with $\left\{\sin \lambda_{n}^{S} z\right\}$ rather than $\left\{\sin \lambda_{n}^{R} z\right\}$ to find, in place of (25),

$$
\sum_{n=1}^{\infty}\left(f_{n}\left(R_{c}\right)-\frac{J_{0}\left(x_{n}\right) s_{n} g_{n}\left(R_{c}\right)}{x_{n} J_{1}\left(x_{n}\right)}\right) a_{j n} C_{n}=b_{j},
$$

with the same $a_{j n}$ 's defined in (27). We have verified numerically that the two procedures gave identical results.

To identify the frequencies of the normal modes we look for the maxima of the pressure field as a function of frequency at a fixed position in space (either inside or outside the column). For the soft-bottom case an alternative 
procedure is to identify the position of the minima of the denominator in Eq. (26). The results obtained by the two methods were identical.

\section{B. The forcing}

The quantity measured experimentally is the sound spectral density SD defined by

$$
\mathrm{SD}=10 \log \frac{4 \pi\left|\hat{P}_{T}(\omega)\right|^{2}}{T\left(P_{\mathrm{ref}}^{2} / \mathrm{Hz}\right)},
$$

where $\hat{P}_{T}$ is the Fourier transform of the measured pressure, $T$ the duration of each sampling interval, and $P_{\text {ref }}$ the reference pressure taken as $1 \mu \mathrm{Pa}$. By virtue of the convolution theorem of the Fourier transform, the spectral response of a linear system (in this case $\hat{P}_{T}$ ) is given by the product of the Fourier transform of the forcing times the complex frequency response, which is the response of the system to an excitation of unit magnitude at frequency $\omega$. The latter is just the quantity $P$ calculated in Sec. II $A$ and, if we denote the former by $\hat{P}_{B}$, we have

$$
\left|\hat{P}_{T}\right|^{2}=\left|\hat{P}_{B}\right|^{2}|P|^{2} \text {. }
$$

As already explained, we assume that $P_{B}$ is due to the process of bubble formation at the base of the column. With the assumption that each such process is independent from the others, the associated acoustic emissions are incoherent and we have

$$
\left|\hat{P}_{B}\right|^{2}=\dot{n} T\left|\left\langle\hat{p}_{B}\right\rangle\right|^{2},
$$

in which $\left\langle\hat{p}_{B}\right\rangle$ denotes the average contribution of each bubble to the excitation of the column and $\dot{n}$ is the number of bubbles generated per unit time given by

$$
\dot{n}=\dot{V} / \frac{4}{3} \pi a^{3},
$$

where $\dot{V}$ is the total volume flow rate of air out of the needles and $a$ the radius of the bubbles. In writing (31) and (32) we have assumed that all the bubbles radiate identically, and that the acoustic emission of each bubble is virtually completed by the end of the sampling time $T$. For $a=1.8 \mathrm{~mm}$ the damping constant is $b \simeq 200 \mathrm{~s}^{-1}$ so that $b T=200 \times 0.1>1$ and this requirement is amply met on average. To close the formulation of the problem it is now necessary to formulate a model for the calculation of $\left\langle p_{B}\right\rangle$.

As air is pushed out of a needle, the surface separating it from the surrounding liquid deforms until, at a certain instant, opposing points come together and a closed surface is formed. The cavity thus produced is not in equilibrium, and it is this initial energy that gives rise to the oscillation of the bubble after it detaches from the needle. At very slow growth rates the initial bubble energy is due to the fact that the surface enclosing the air pocket does not have the equilibrium spherical shape. At the higher growth rates of this experiment, however, a much greater amount of energy is stored as kinetic energy in the liquid.

We assume that, as can be verified a posteriori, the bubbles oscillate linearly with the natural frequency $\omega_{0}$ and damping parameter $b$ introduced before in Eq. (3), and we write

$$
R(t)=a\left[1+X_{0} \exp (-b t) \sin \left(\omega_{0} t+\psi\right)\right],
$$

where $X_{0}$ is the initial amplitude of oscillation and $\psi$ is the initial phase. Since the initial kinetic energy is much greater than the initial potential energy due to the distortion of the spherical shape, $\psi$ is small and we therefore take $\psi=0$. If the system consists of $N$ needles, the initial radial expansion velocity $\dot{R}(0)$ of the bubble wall when the bubble detaches from the needle is given approximately by (Strasberg, 1956)

$$
4 \pi N a^{2} \dot{R}(0)=\dot{V} .
$$

By substituting the time derivative of (33) at $t=0$ into this relation we find

$$
X_{0}=\dot{V} / 4 \pi N a^{3} \omega_{0}=\dot{n}_{N} / 3 \omega_{0} .
$$

In the experiment typical values are $\dot{n}_{N} \sim 50 \mathrm{~s}^{-1}$ and $\omega_{0} \simeq 2 \pi \times 1900 \mathrm{~s}^{-1}$ so that $X_{0} \simeq 0.001$, which is indeed small enough to justify the hypothesis of linear oscillations.

The considerations of this subsection have so far been focused on the acoustic emission of the individual bubbles, while the model of the bubbly column described in Sec. II $B$ is an average one in which the bubbles' individuality is disregarded. We must establish a connection between the two points of view so as to determine the effectively uniform forcing at the base of the column assumed in the previous analysis.

The pressure field at a distance $r$ from the center of an isolated bubble is

$$
p_{B}=\rho\left(a^{2} / r\right) \ddot{R} .
$$

The base of the column of bubbly liquid has an area $\pi R_{c}^{2}$. We associate to each one of the $N$ needles the area $A_{n}=\pi R_{c}^{2} / N$ and average (36) over this area to find

$$
\bar{p}_{B}=\frac{1}{A_{n}} \int p_{B} d A_{n}=2 \rho \sqrt{N} \frac{a^{2}}{R_{c}} \ddot{R} .
$$

The production noise due to one "generation" of $N$ bubbles (one per needle) at the base of the column would be necessary to give rise to this pressure level. The contribution of just one bubble, which is the quantity needed in Eq. (31), is therefore

$$
\left|\left\langle\hat{p}_{B}\right\rangle\right|^{2}=(1 / N)\left|\hat{\bar{p}}_{B}\right|^{2},
$$

so that

$$
\left|\hat{P}_{B}\right|^{2}=4 \rho^{2} \dot{n} T\left(a^{4} / R_{c}^{2}\right)|\hat{\ddot{R}}|^{2} .
$$

A simple interpretation of the argument used to obtain this result is that the base of the column has been divided into $N$ incoherent "pistons," each one of which acts on the column with a pressure equal to the (37) over the area $\pi R_{c}^{2} / N$.

An alternative derivation of the same result is as follows. Suppose that the bubbles are separated in time so that each one has essentially reached equilibrium by the time another one is produced. With a damping constant of the order of $500 \mathrm{~s}^{-1}$ and a bubble frequency of the order of $50 / \mathrm{s}$ this condition is approximately verified. The average 


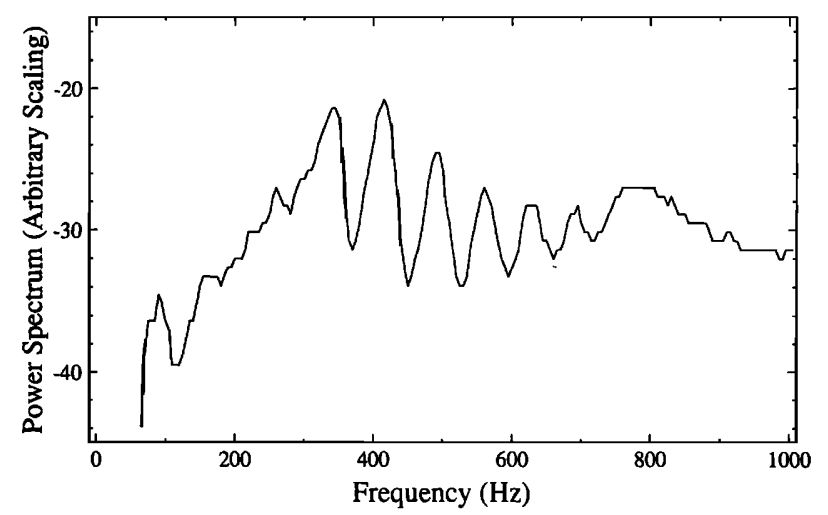

FIG. 4. Example of the hydrophone output (log scale) for the softbottom case. The hydrophone was positioned $0.170 \mathrm{~m}$ from the axis of the column at a depth of $0.110 \mathrm{~m}$. The bubble volume fraction was $0.70 \%$.

pressure to be associated with each bubble must now be calculated by using the entire column cross-sectional surface and is

$$
\left\langle p_{b}\right\rangle=2 \rho\left(a^{2} / R_{c}\right) \ddot{R},
$$

which can be substituted directly into (31) to find the same result (39).

To calculate $\hat{\ddot{R}}$ we take $R=a$ for $t<0$ and use (33) for $t>0$ to find

$$
|\hat{\ddot{R}}|^{2}=\frac{X_{0}^{2} a^{2} \omega_{0}^{2}}{2 \pi} \frac{\omega_{0}^{4}+b^{2}\left(2 \omega_{0}^{2}+4 \omega^{2}+b^{2}\right)}{\left(b^{2}-\omega^{2}+\omega_{0}^{2}\right)^{2}+4 \omega^{2} b^{2}} .
$$

At frequencies much below the resonance frequency the second fraction is approximately 1 and the forcing pressure amplitude is therefore approximately constant. For $a=1.8$ $\mathrm{mm}$ the resonant frequency is $\omega_{0} / 2 \pi=1883 \mathrm{kHz}$. The damping parameter $b$ is a function of frequency. It equals $197 \mathrm{~s}^{-1}$ at resonance and $\approx 1000 \mathrm{~s}^{-1}$ at $400 \mathrm{~Hz}$.

\section{RESULTS}

We show in Fig. 4 a typical example of the power spectrum of the hydrophone output as displayed on the oscilloscope. The several peaks are interpreted as corresponding to the normal modes of the system, and we wish to compare their level and location with those predicted by the theory outlined in the previous section. Before we do so, however, it is useful to recall the points in which the mathematical model deviates from the experimental system.

(i) The outer boundary of the experimental set-up is square, while the mathematical model postulates a circular shape. Hence, in the comparison, we do not expect close agreement for those modes that are not exponentially attenuated outside the column, but retain an appreciable amplitude near the outer boundary. These modes can be identified from the theoretical results. Furthermore, the value to be assumed in the theory for the radius of the outer boundary is somewhat arbitrary and could range between $0.49 \mathrm{~m}$-i.e., one-half the side of the cubical tank-and $0.71 \mathrm{~m} \longrightarrow$ one-half the diagonal of the cube's faces. (ii) For the rigid-bottom case the height of the bubbly column is unambiguously defined because the needle tips are flush with the concrete slab. For the soft-bottom case, however, the situation is not so clear-cut. The needles are mounted on a rectangular metal plate so that their tips are $0.102 \mathrm{~m}$ above the tank's bottom and $0.820 \mathrm{~m}$ below the water level (the experimental error in these data is judged to be $\pm 2 \mathrm{~mm}$ ). Any value of $h$ between 0.820 and $0.925 \mathrm{~m}$ can therefore in principle be justified.

(iii) The pressure-release boundary condition assumed at the external boundary and at the base of the tank is an approximation of unknown accuracy, which is bound to get worse as the frequency of the modes-and therefore their order-increases.

(iv) The model assumes a monodisperse bubble cloud, while in reality the bubble diameters spans a finite (if small) range. The bubble-size effect is negligible at frequencies much lower than the natural frequencies of the bubbles, but increasingly less so with increasing frequency. Furthermore, albeit small, there is an effect of the hydrostatic pressure as the bubbles rise from the needles to the surface.

(v) As was mentioned earlier, the bubbles at the periphery of the column rise with a velocity that is about $14 \%$ smaller than those at the center. Since the airflow into each needle is approximately the same, this means that the column has a slightly higher void fraction at its boundary compared to the core.

(vi) The excitation model for the bubble column is clearly a simplification of the actual physical situation.

(vii) The background noise level in the experiment was around 60-70 dB. Any theoretical prediction below this level cannot therefore be compared with the data.

It is against the backdrop of these differences between physical system and mathematical model that the comparison that follows must be judged. Hence, rather than on the agreement or disagreement of individual theoretical predictions and experiment, the focus should be on the overall consistency and experimental validation of the interpretation of the data that emerges from this study.

\section{A. Soft bottom}

Figure 5 shows a graph of the first six modes as measured (symbols) and calculated theoretically (lines) as a function of the gas volume fraction. For these results a bubble radius of $a=1.8 \mathrm{~mm}$, a tank radius of $R_{t}=0.71 \mathrm{~m}$ (equal to one-half of the diagonal of the tank's square platform), and column height of $h=0.87 \mathrm{~m}$ (from the free surface to the base of the needles) were assumed in the theory. There is generally a good agreement between theory and experiment, which however deteriorates somewhat at the lowest void fractions. This feature is probably a consequence of the fact that here the frequency of the normal modes becomes closer to that of the bubbles, a region where the effective-medium model for the bubbly mixture used here is known from previous work not to be as accurate (Commander and Prosperetti, 1989). Since the actual height of the bubbly region is $0.820 \mathrm{~m}$, rather than $0.87 \mathrm{~m}$, to generate the previous figure the value of $\beta$ was adjusted 


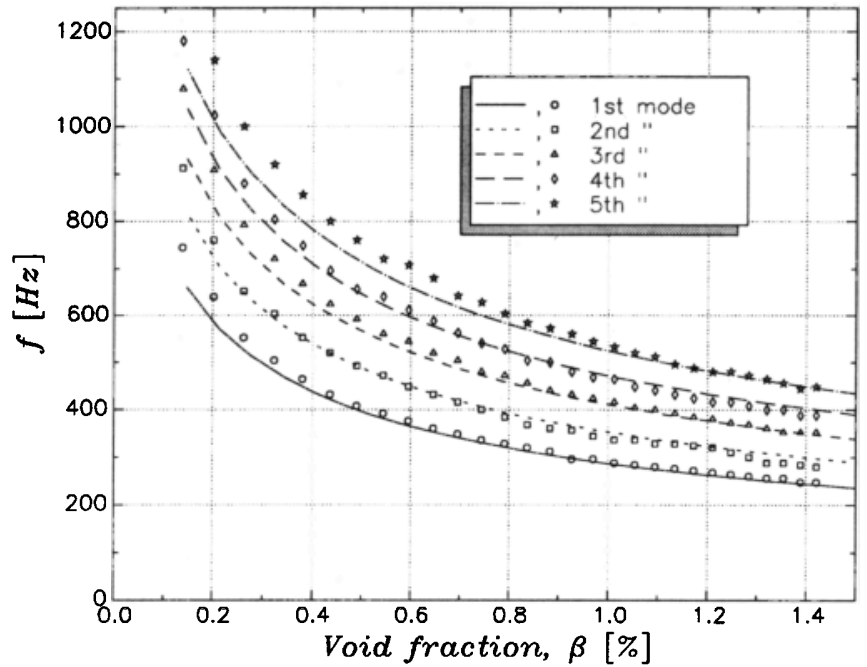

FIG. 5. Graph of the lowest five modes for the soft-bottom case as a function of the volume fraction. The symbols are the data and the lines the theoretical results. The experimental precision is estimated to be $\pm 10 \mathrm{~Hz}$.

so as to keep the same gas volume in the bubble cloud. Furthermore, the equilibrium pressure was assumed to correspond to the hydrostatic pressure halfway through the tank. If both these adjustments are removed, the comparison deteriorates somewhat with the theoretical lines moving more and more toward lower frequencies as the void fraction is decreased and the mode number increased. For the fifth mode and $\beta=0.20 \%$ the difference is of the order of $4.3 \%$, and for $\beta=1.00 \%$ of the order of $4 \%$. For all the following results the adjusted values of the hydrostatic pressure and gas volume fraction have been used.

In the course of the experiment it was noticed that, as the hydrophone was moved vertically toward the bottom of the tank, the position of the resonance peaks shifted by

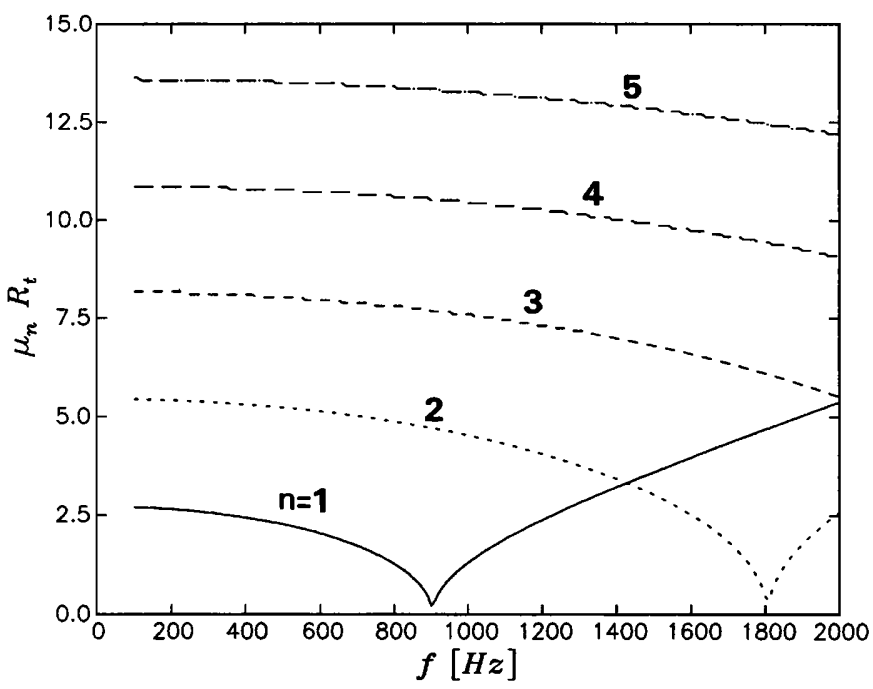

FIG. 6. Modulus of the quantity $\mu_{n} R_{t}$ defined by (14) as a function of frequency for the different modes. Here, $\mu_{n}$ is pure imaginary to the left of the zeros and real to the right. $\exp \left[-\left(\mathscr{T} \mu_{n} R_{t}\right)\right]$ may be interpreted as the ratio between the pressure amplitude on the column axis and at the tank wall. Note how the higher modes are progressively more attenuated.
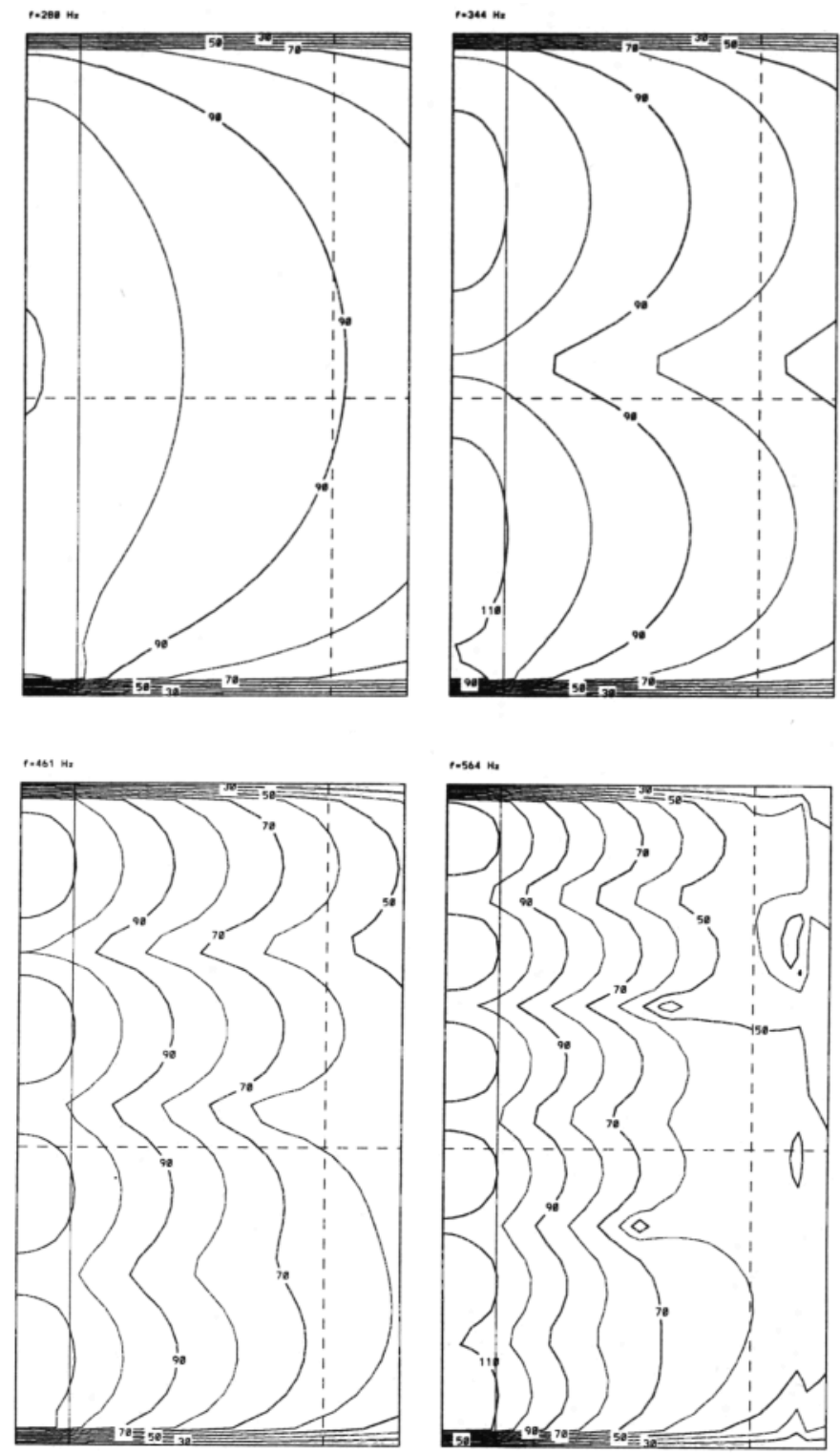

FIG. 7. Theoretical pressure distributions (in units of $\mathrm{dB}$ re: 1 $\left.\mu \mathrm{Pa} / \mathrm{Hz}^{1 / 2}\right)$ in the tank in correspondence of the first, second, fourth, and sixth eigenmodes for $\beta=1.05 \%$. The left vertical boundary is the axis of the bubble column. The bubbly liquid edge and the positions for which the different modes are plotted are marked by a solid line and dashed lines, respectively. The width and height of each frame are 0.500 and $0.820 \mathrm{~m}$, respectively.

10-20 Hz. We estimate the data shown in this figure to be affected by an error of this order. These points are based on hydrophone readings taken midway down the column, near its outer edge in the pure liquid region.

We have also explored the effect of the bubble size on the predicted eigenfrequencies. Going from 1.8 to $1 \mathrm{~mm}$ has a completely negligible effect on the first mode while the frequency of the fifth mode is left unchanged for $\beta \geqslant 1 \%$, but gradually rises for smaller volume fractions by as much as $10 \%$ at $\beta=0.2 \%$.

A variation in the tank radius from 0.5 to $0.71 \mathrm{~m}$ has a very small effect, which gets weaker as the order of the mode grows. The maximum difference for mode 1 is of the order of $1 \%$. If the nature of the tank wall is changed from soft to rigid, again the higher modes are unchanged while 
(o)

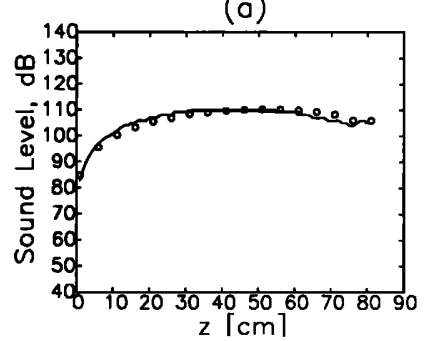

(c)
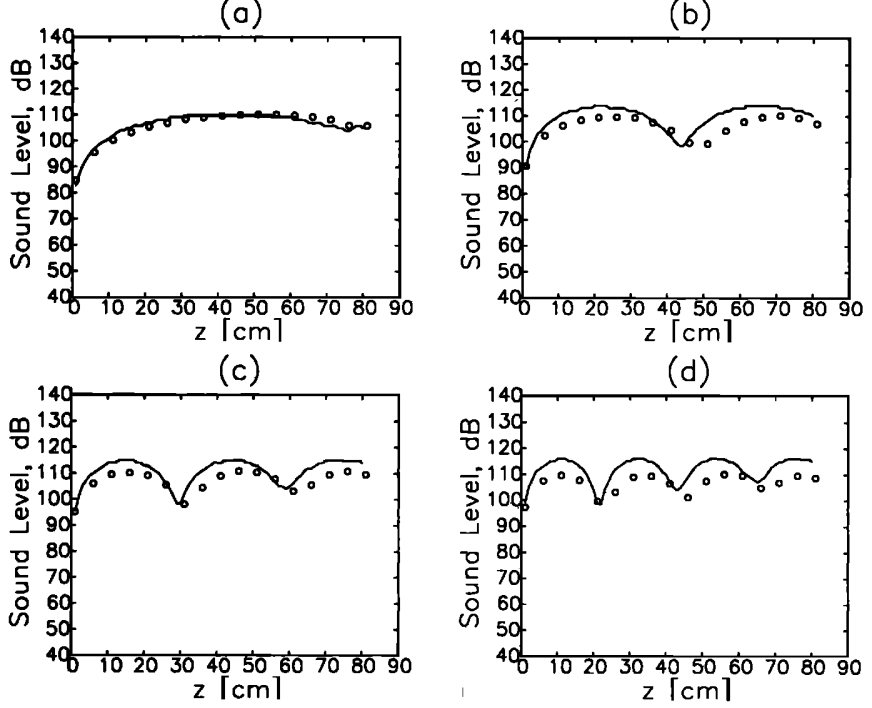

(d)

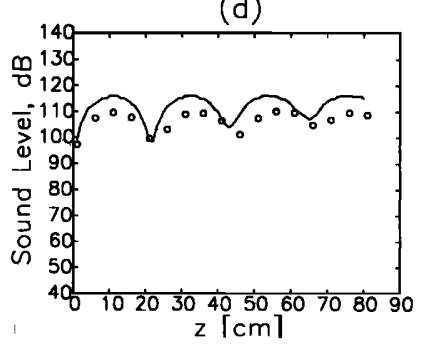

FIG. 8. Comparison between measurements (circles) and theoretical results for the spectral density distribution in the lowest four modes along the axis of the bubble column with $\beta=1.05 \%$ in units of $\mathrm{dB}$ re: 1 $\mu \mathrm{Pa} / \mathrm{Hz}^{1 / 2}$. The experimental and theoretical frequencies are, respectively, (a) 290 and $283 \mathrm{~Hz}$; (b) 345 and $346 \mathrm{~Hz}$; (c) 400 and $402 \mathrm{~Hz}$; (d) 455 and $461 \mathrm{~Hz}$.

the first mode eigenfrequencies drops by a maximum of about $9.7 \%$. To understand this lack of sensitivity to the position and nature of the tank's wall it is useful to consider Fig. 6, which shows the modulus of the quantity $\mu_{n} R_{t}$ defined in (14) for $n=1, \ldots, 5$ as a function of frequency. In the portion of the curves to the left of the zeros, $\mu_{n}$ is imaginary, and $\exp \left[-\left(\Im \mu_{n} R_{t}\right)\right]$ may be interpreted as the ratio between the amplitude on the column axis and at the tank wall. To the right of the zeros the exponentials are purely imaginary and there is only cylindrical spreading. It is seen that, over the entire range of frequencies depicted in Fig. 5, the modes are exponentially attenuated with a higher and higher rate as the mode order increases. Hence, one expects a somewhat higher sensitivity to the tank boundary for the lower modes.

We now examine in greater detail the pressure levels and distribution in the system. Unless otherwise stated, all of the calculations have been done for the same conditions as Fig. 5 above. An overall picture of the sound field is given in Fig. 7, which shows the calculated intensity contour plots (in $\mathrm{dB}$ re: $1 \mu \mathrm{Pa} / \mathrm{Hz}^{1 / 2}$ ) for the first, second, fourth, and sixth normal modes at $\beta=1.05 \%$. In agreement with Fig. 6 the lowest modes are seen to propagate farther from the axis, while the sound field of the higher ones is strongly localized near the bubble column.

Figures 8 and 9 compare the predicted and measured vertical pressure distributions of the first four modes along the column axis, $r=0$, and along the line $r=0.4 \mathrm{~m}$. The theoretical curves are plotted for the theoretical mode frequencies, which in some cases are slightly different from the experimental ones as indicated in the figure captions. The experimental frequencies were set near the top of the column and are therefore slightly different from those plotted in Fig. 5, which are based on midheight readings. Each (o)

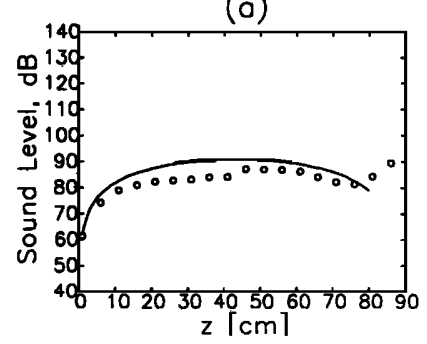

(c)
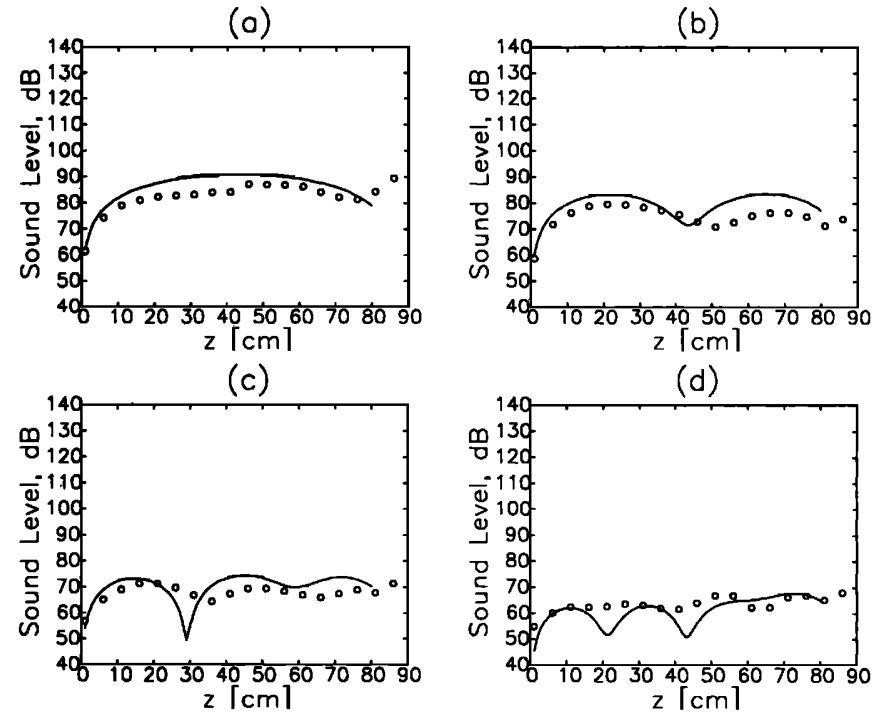

(d)

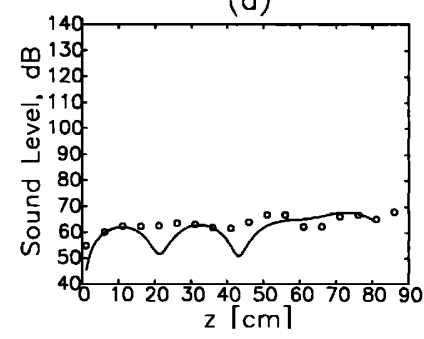

FIG. 9. Same as Fig. 8 along a vertical line at a distance of $0.4 \mathrm{~m}$ from the column axis. The background noise level was $60-70 \mathrm{~dB}$ re: $1 \mu \mathrm{Pa} / \mathrm{Hz}^{1 / 2}$ depending on the frequency.

experimental data point is the average of 500 to 1000 readings. Since the peak position underwent slight fluctuations during the experiment, one expects that the averaging will result in some underestimation of the peak pressure.

Inside the column (Fig. 8), there is a general consistency of the profile shape and sound level. The level matches the data for the lowest mode, but the comparison gets progressively worse as the mode number increases. The wavelengths of the modes appear to be underpredicted by the theory, but this is a consequence of the use of an "effective" depth of $0.875 \mathrm{~m}$. If the depth is set at the actual tank depth of $0.925 \mathrm{~m}$ (Fig. 10), the theoretical wavelength becomes much closer to the experimental one. In spite of this result, we continue to use the depth of 0.875 $\mathrm{m}$ for consistency with the mode frequencies of Fig. 5 . Analogous comments apply to the results shown in Fig. 9.

Similar comparisons for the sound distribution in the horizontal plane $z=-0.48 \mathrm{~m}$ are shown in Fig. 11. The data shown here and those of a similar nature discussed later were taken along a line perpendicular to the sides of the tank and passing through the column's axis, while the theoretical calculations are carried out for a greater hori-

(o)

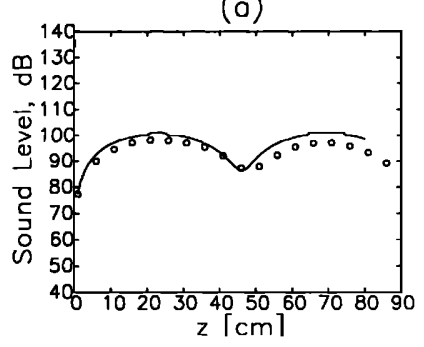

(b)

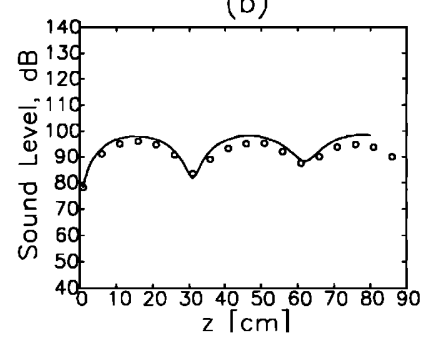

FIG. 10. Effect of column height assumed in the theory on the spectral density distribution along the column axis for the second and third mode. The experimental and theoretical frequencies are, respectively, (a) 345 and $346 \mathrm{~Hz}$; (b) 400 and $402 \mathrm{~Hz}$. 
(a)

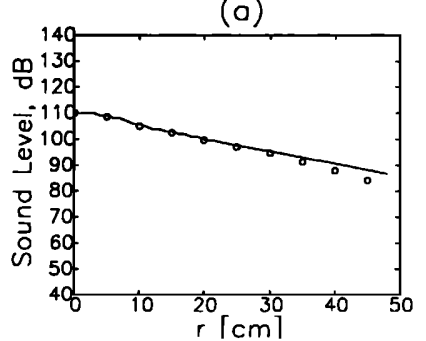

(c)
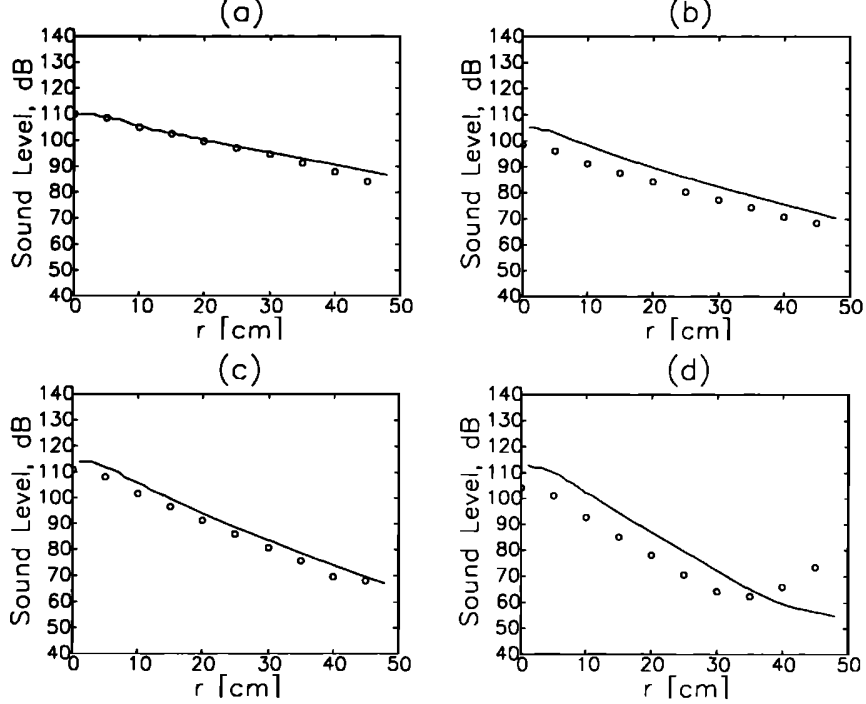

(d)

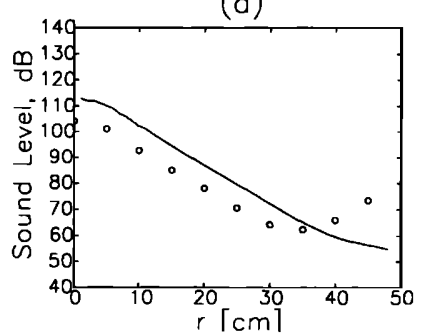

FIG. 11. Comparison between measurements (circles) and theoretical results for the spectral density distribution corresponding to the lowest four modes in a plane at a depth of $0.480 \mathrm{~m}$ with $\beta=1.05 \%$. The background noise level was $60-70 \mathrm{~dB}$ re: $1 \mu \mathrm{Pa} / \mathrm{Hz}^{1 / 2}$ depending on the frequency.

zontal dimension, $R_{t}=0.71 \mathrm{~m}$. While the effect of this discrepancy will not be large because of the attenuation of the sound field away from the bubbly region, some differences near the outer boundary of the tank may be expected and are indeed apparent from the figures. The sound level matches the data well for the lowest mode, but overestimates them for higher modes as before. The noise "floor" at $60-70 \mathrm{~dB}$ affects this comparison.

Figures 12 and 13 show similar results for a smaller gas volume fraction, $\beta=0.70 \%$. The point we wish to stress with these figures is that the results found previously for $\beta=1.05 \%$ are quite typical. At the lower gas volume fraction, on average, there is a somewhat closer match between theory and experiment, but the same trends are present. In particular, agreement with the measured wavelengths improves with the use of $h=0.925 \mathrm{~m}$. In this case, much of the higher-mode data is below the background noise level. (a)

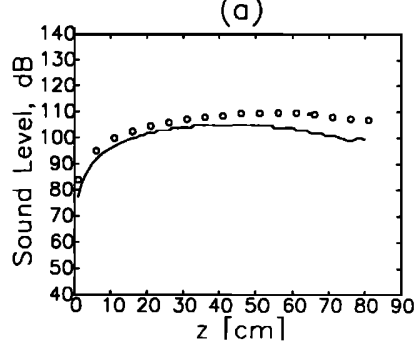

(b)

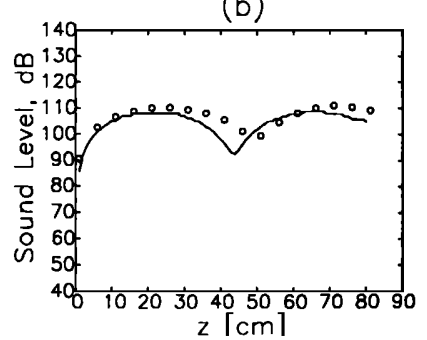

FIG. 12. Comparison between measurements (circles) and theoretical results for the spectral density distribution in the lowest two modes along the axis of the bubble column with $\beta=0.70 \%$. The experimental and theoretical frequencies are, respectively, (a) 350 and $344 \mathrm{~Hz}$; (b) 420 and $421 \mathrm{~Hz}$. (o)
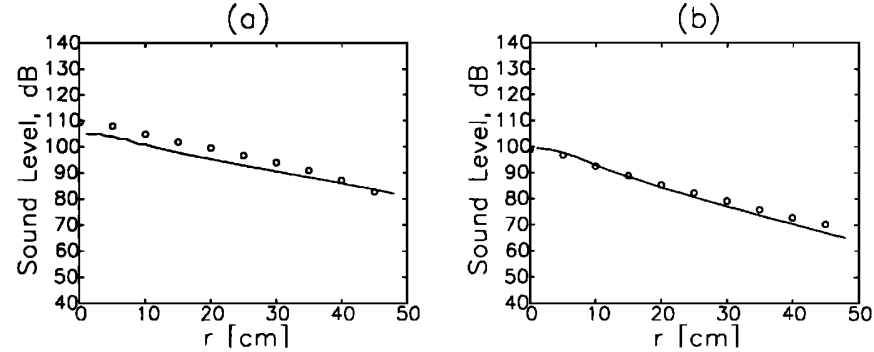

FIG. 13. Comparison between measurements (circles) and theoretical results for the spectral density distribution corresponding to the lowest two modes in a plane at a depth of $0.480 \mathrm{~m}$ with $\beta=0.70 \%$. The background noise level was $60-70 \mathrm{~dB}$ depending on the frequency.

\section{B. Concrete bottom}

The experimental readings were more difficult when the concrete slab was inserted into the tank because the system was found to be much noisier than before. Figure 14 shows a comparison between the measured and computed eigenfrequencies of the system in this case. As explained before, no adjustment of the column height and volume fraction is now necessary. Agreement between theory and experiment is, however, not as good as the one found previously, particularly for the lowest mode and particularly at low volume fractions. An important effect that may account at least in part for this difference can be illustrated with reference to Fig. 15 which is analogous to the previous Fig. 6 and shows the dimensionless wave number $\mu_{n} R_{t}$ given by (14) as a function of frequency for the different modes. It will be recalled that in the range to the left of the zeros, $\exp \left[-\left(\Im \mu_{n} R_{t}\right)\right]$ may be interpreted as the ratio between the amplitude on the column axis and at the tank wall. It is seen that, for the lowest mode, this ratio is relatively large: the modes propagate appreciably far from the column, the details of the outer boundary are important, and the mismatch between the model's geome-

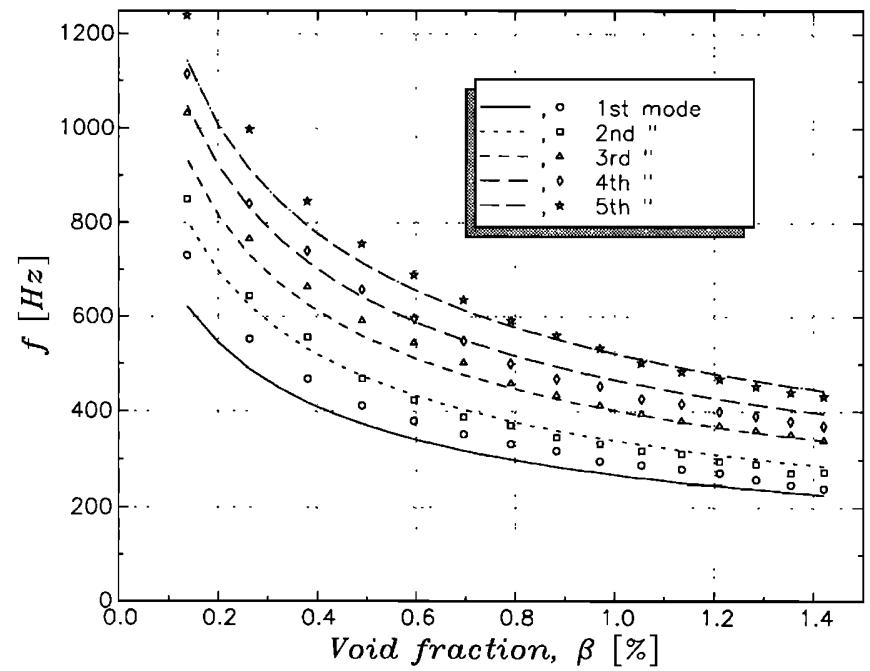

FIG. 14. Graph of the lowest five modes for the hard-bottom case as a function of the volume fraction. The symbols are the data and the lines the theoretical results. The experimental precision is estimated to be $\pm 10 \mathrm{~Hz}$. 


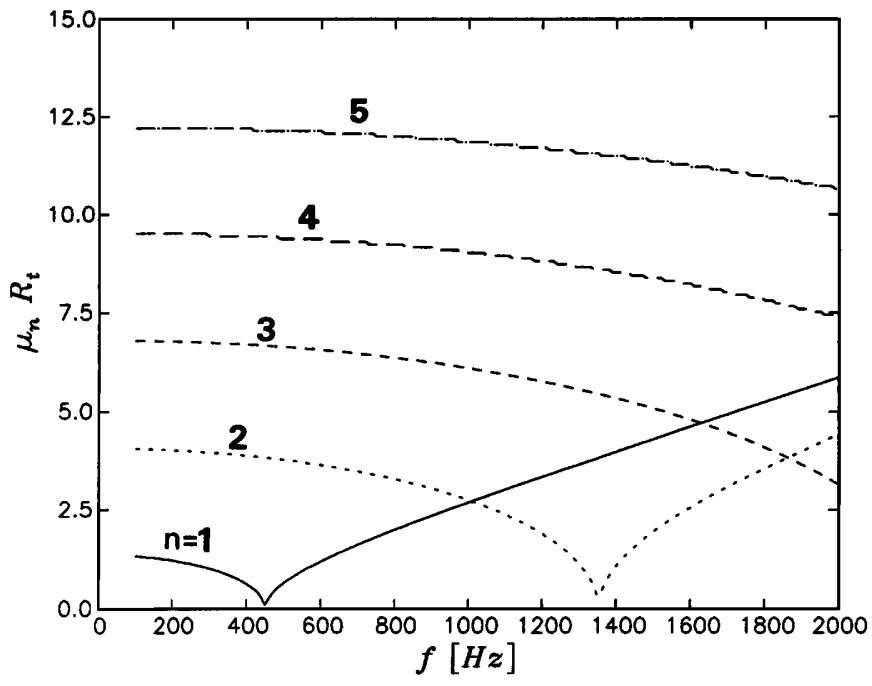

FIG. 15. Modulus of the quantity $\mu_{n} R_{t}$ defined by (14) as a function of frequency for the different hard-bottom modes. $\mu_{n}$ is pure imaginary to the left of the zeros and real to the right. $\exp \left[-\left(\Im \mu_{n} R_{t}\right)\right]$ may be interpreted as the ratio between the pressure amplitude on the column axis and at the tank wall. Note that the radial attenuation is much smaller than in the soft-bottom case.

try and the actual one affects strongly (and negatively) the comparison. The attenuation of the higher modes with distance from the column axis is stronger and therefore the comparison is more favorable. The problem with the lower volume fractions is the same as before, namely the mode's frequency getting close to the resonant frequency of the individual bubbles.

Figure 16 shows a computed two-dimensional "map"

1. $262 . \mathrm{Hz}$
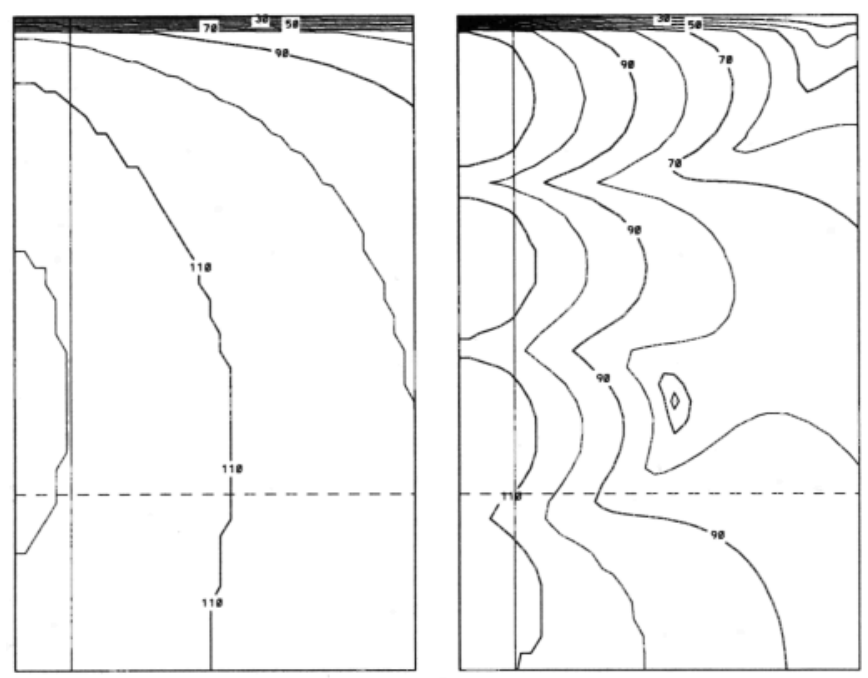

FIG. 16. Theoretical spectral density distributions in the tank in correspondence with the first and fourth eigenmodes for $\beta=1.05 \%$ and rigidbottom boundary conditions. The left vertical boundary is the axis of the bubble column. The bubbly liquid edge and the positions for which the different modes are plotted are marked by a solid and a dashed line, respectively. The width and height of each frame are 0.50 and $0.820 \mathrm{~m}$, respectively. (a)
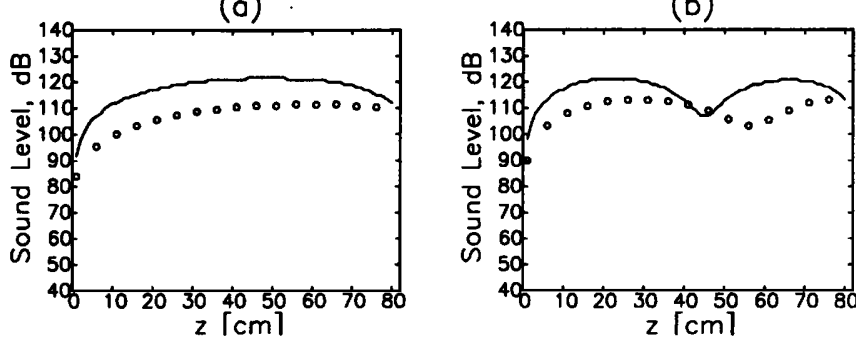

FIG. 17. Comparison between measurements (circles) and theoretical results for the spectral density distribution in the lowest two hard-bottom modes along the axis of the bubble column with $\beta=1.05 \%$. The experimental and theoretical frequencies are, respectively, (a) 282 and $262 \mathrm{~Hz}$; (b) 331 and $331 \mathrm{~Hz}$. The mathematical model in these cases does not correspond closely to the experiment. See text.

of the sound field similar to that of Fig. 6 of the softbottom case for the first and fourth modes.

Some examples of a comparison of the measured and calculated sound fields of the single modes are shown in Figs. 17 and 18. Generally speaking, the situation is considerably worse than in the soft-bottom case. Figure 17 shows the sound intensity as a function of depth along the axis of the column for the first two modes. The theoretical levels are generally higher and the computed wavelength does not match the experimental one. A similar picture emerges from Fig. 18 where the sound level distribution on the horizontal plane $z=-0.600 \mathrm{~m}$ is shown for the first four modes.

We believe that this situation is the result of an insufficient rigidity of the concrete slab which fails to provide a true rigid-bottom boundary condition. To prove this statement, recall the expression for the lowest natural frequency (a)

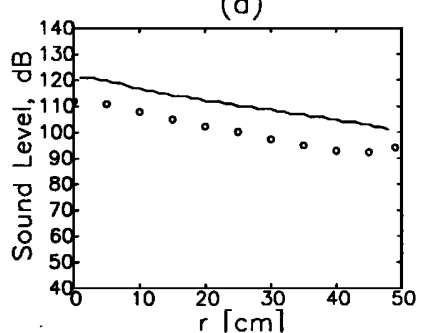

(c)

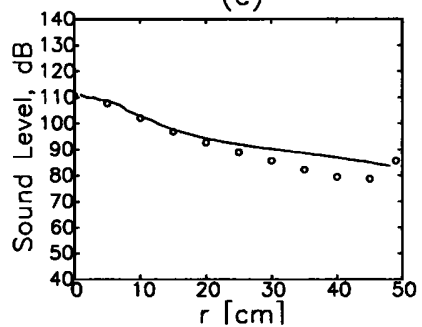

(b)

(d)

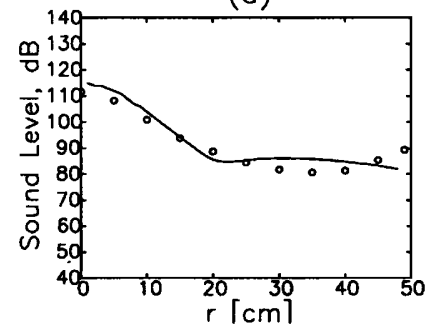

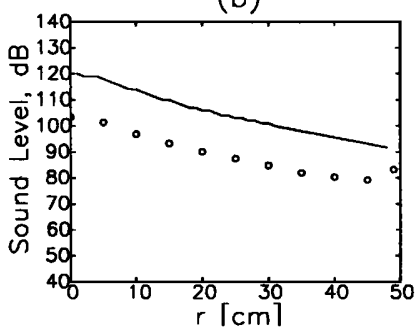

FIG. 18. Comparison between measurements (circles) and theoretical results for the spectral density distribution corresponding to the lowest four hard-bottom modes in a plane at a depth of $0.600 \mathrm{~m}$ with $\beta=1.05 \%$. The experimental and theoretical frequencies are, respectively, (a) 282 and $262 \mathrm{~Hz}$; (b) 331 and $331 \mathrm{~Hz}$; (c) 386 and 392 Hz; (d) 494 and 455 Hz. The mathematical model in these cases does not correspond closely to the experiment. See text. 
$v_{S}$ of a square slab of thickness $H$ clamped along the edges (see, e.g., Landau and Lifshitz, 1959)

$$
v_{S}=\left(\frac{E}{\rho_{C}\left(1-v_{P}^{2}\right)}\right)^{1 / 2} \frac{\pi H}{A^{2}} .
$$

Here $E$ is Young's modulus, $v_{P}$ is the Poisson ratio, $\rho_{C}$ is the density of the material, and $A$ the length of the side. One can assume the slab to behave rigidly only if $v_{S}$ is much greater than the frequencies of interest. Upon taking $E=21 \mathrm{GPa}, v_{P}=0.15$, and $\rho_{C}=2400 \mathrm{~kg}$, all of which are reasonable values for concrete, with $H=0.05 \mathrm{~m}$, one finds $v_{S} \simeq 136 / A^{2}$. With $A \simeq 0.5 \mathrm{~m}$ (an average distance between the wooden beams supporting the tank on the floor), $v_{S}=543 \mathrm{~Hz}$. A direct measurement of the slab resonances gave approximately 580 and $720 \mathrm{~Hz}$. Hence, far from a rigid boundary, one is really dealing with a very complex coupled system in which the modes of the fluid and the concrete couple and interact. In this situation not much more than a qualitative agreement between theory and experiment can be expected, and indeed such is the conclusion that can be drawn from Figs. 17 and 18. In particular, we wish to draw the reader's attention to the fact that once again, as in the soft-bottom case, the excitation mechanism of the system based on the free oscillations of the departing bubbles seems to be able to roughly account for the measured levels.

Since the model and the data correspond really to two different situations, we do not include a more detailed discussion of this case.

\section{CONCLUSIONS}

We have presented a comparison between data and theory for the system shown in Fig. 1. While the mathematical model departs in several respects from the physical system so that an exact correspondence cannot be expected, the results lend a strong support to the theory itself and to the conceptual model on which it is based.

The theory of the acoustic behavior of the bubbly mixture rising at the center of the tank had been presented earlier and shown to agree with the data for the fundamental mode. The results of this paper complete and strengthen the earlier ones. Furthermore, we have presented an analysis of the manner in which the normal modes of the system are excited. We have postulated that this excitation is due to the free oscillations of the bubbles as they depart from the needles. In spite of the fact that these oscillations occur at frequencies of the order of 3-6 times the normal modes of the system, we have found that the absolute noise levels predicted on the basis of this hypothesis match the data. This is due to the nonzero width of the single-bubble spectrum caused by damping.

This study had been undertaken to examine in a laboratory setting the validity of the hypothesis that bubble clouds at the ocean surface could be responsible for lowfrequency oceanic ambient noise. We have shown that lowlevel normal modes exist and that they can be excited by the mechanism by which the individual bubble are formed. This conclusion lends support to the previous hypothesis.
In a related paper (Oguz, 1994), on the basis of an approach similar to the present one, oceanic ambient noise is estimated with very good agreement with the data both in terms of spectrum and levels. Those results are found to be quite robust with respect to details of the actual nature of the breaking process and attendant air entrainment such as void fraction, extent in space and time, bubble radius, and number.

\section{ACKNOWLEDGMENTS}

One of the authors (M.N.) would like to thank Ken Markiewicz for his help in collecting and reducing some of the experimental data.

This work has been supported by the Ocean Acoustic Program of ONR.

Carey, W. M., and Bradley, M. P. (1985). "Low-frequency ocean surface noise sources,” J. Acoust. Soc. Am. Suppl. 1 78, S1-S2.

Carey, W. M., and Browning, D. G. (1988). "Low-frequency ocean ambient noise: Measurements and theory," in Sea Surface Sound, edited by B. R. Kerman (Kluwer, Dordrecht), pp. 361-376.

Carey, W. M., Fitzgerald, J. W., and Browning, D. G. (1993). "Lowfrequency noise from breaking waves," in Natural Physical Sources of Underwater Sound, edited by B. R. Kerman (Kluwer, Dordrecht), pp. 277-304.

Carey, W. M., and Wagstaff, R. A. (1986). "Low-frequency noise fields," J. Acoust. Soc. Am. 80, 1523-2526.

Commander, K. W., and Prosperetti, A. (1989). "Linear pressure waves in bubbly liquids: Comparison between theory and experiments," $J$. Acoust. Soc. Am. 85, 732-746.

Kennedy, R. M. (1992). "Sea surface dipole sound source dependence on wave-breaking variables,” J. Acoust. Soc. Am. 91, 1974-1982.

Kennedy, R. M., and Goodnow, T. V. (1990). "Measuring the vertical directional spectra caused by sea surface sound," IEEE J. Ocean Eng. 15, 299-310.

Kerman, B. R. (1984). "Underwater sound generation by breaking waves,” J. Acoust. Soc. Am. 75, 149-165.

Kewley, D. J., Browning, D. G., and Carey, W. M. (1990). "Lowfrequency wind-generated ambient noise source levels," J. Acoust. Soc. Am. 88, $1894-1902$.

Kuperman, W. A., and Ferla, M. C. (1985). "A shallow-water experiment to determine the source spectrum level of wind-generated noise," J. Acoust. Soc. Am. 77, 2067-2073.

Koller, D. P., and Shankar, P. M. (1993). "Low-frequency oscillations of bubble plumes,” J. Acoust. Soc. Am. 93, 1362-1364.

Landau, L., and Lifshitz, E. (1959). Theory of Elasticity (translated from Russian by J. B. Sykes and W. H. Reid) (Pergamon, New York), Sec. 25.

Lu, N. Q., and Prosperetti, A. (1993). "Active and passive acoustic behavior of bubbly layers," J. Acoust Soc. Am., submitted.

Lu, N. Q., Prosperetti, A., and Yoon, S. W. (1990). "Underwater noise emissions from bubble clouds," IEEE J. Ocean Eng. 15, 275-281.

Monahan, E. C., and Lu, M. (1990). "Acoustically relevant bubble assemblages and their dependence on meteorological parameters," IEEE J. Ocean Eng. 15, 340-349.

Monahan, E. C., and Mac Niocaill, G., Eds. (1986). Oceanic Whitecaps (Reidel, Dordrecht).

Õguz, H. N. (1994) "A theoretical study of low frequency oceanic ambient noise," J. Acoust. Soc. Am. 95, 1895-1912.

Perrone, A. J. (1969). "Deep ocean ambient noise spectra in the Northwest Atlantic," J. Acoust. Soc. Am. 46, 762-770.

Phillips, O. M. (1988). "Equilibrium range characteristics of breaking waves," in Sea Surface Sound, edited by B. R. Kerman (1988) (Kluwer, Dordrecht), pp. 31-38.

Piggott, C. L. (1964). "Ambient sea noise at low frequencies in shallow water of Scotian shelf," J. Acoust. Soc. Am. 36, 2152-2163.

Prosperetti, A. (1985). "Bubble-related ambient noise in the ocean," J. Acoust. Soc. Am. Suppl. 1 78, S2. 
Prosperetti, A. (1988a). "Bubble dynamics in oceanic ambient noise," in Sea Surface Sound, edited by B. R. Kerman (Kluwer, Dordrecht), pp. 151-171.

Prosperetti, A. (1988b). "Bubble-related ambient noise in the ocean," J. Acoust. Soc. Am. 84, 1042-1054.

Prosperetti, A., Lu, N. Q., and Kim, S. H. (1993). "Active and passive acoustic behavior of bubble clouds at the ocean's surface," J. Acoust Soc. Am. 93, 3117-3127.

Sarkar, K., and Prosperetti, A. (1993). "Backscattering of underwater noise by bubble clouds," J. Acoust Soc. Am. 93, 3128-3138.

Strasberg, M. (1956). "Gas bubbles as sources of sound in liquids," J. Acoust. Soc. Am. 28, 20-26.

Thorpe, S. A. (1982). "On the clouds of bubbles formed by breaking wind waves in deep water and their role in air-sea gas transfer," Philos. Trans. R. Soc. London Ser. A 304, 155-210.

Thorpe, S. A. (1986). "Measurements with an automatically recording inverted echo sounder: Aries and bubble clouds," J. Phys. Oceanogr. 16, 1462-1478.

Thorpe, S. A., and Hall, A. J. (1983). "The characteristics of breaking waves, bubble clouds, and near-surface currents observed using sidescan sonar," Continental Shelf Res. 1, 353-384.

Toba, Y., and Koga, M. (1986). "A parameter describing overall conditions of wave breaking, whitecapping, sea-spray production, and wind stress," in Oceanic Whitecaps, edited by E. C. Monahan and G. Mac Niocaill (Reidel, Dordrecht), pp. 37-47.

Wenz, G. M. (1962). "Acoustic ambient noise in the ocean: Spectra and sources," J. Acoust. Soc. Am. 34, 1936-1956.

Wille, P. C., and Geyer, D. (1985). "Measurements on the origin of the wind-dependent ambient noise variability in shallow water," J. Acoust. Soc. Am. 75, 173-185.

Wilson, J. J. (1980). "Low-frequency wind-generated noise produced by the impact of spray with the ocean's surface," J. Acoust. Soc. Am. 68, 952-956.

Wu, J. (1988). "Variations of whitecap coverage with wind stress and water temperature," J. Phys. Oceanogr. 18, 1448-1453.

Yoon, S. W., Crum, L. A., Prosperetti, A., and Lu, N. Q. (1991). An investigation of the collective oscillations of a bubble cloud," J. Acoust. Soc. Am. 89, 700-706. 